\title{
Iris Recognition System
}

\author{
Neha Kak, Rishi Gupta \\ Computer Science Engineering \\ Lingayas Institute of Technology and Management \\ Faridabad, Haryana,INDIA \\ neha_kak@yahoo.in, rishi26gupta@gmail.com
}

\author{
Sanchit Mahajan \\ Information Technology \\ Lingayas Institute of Technology and Management \\ Faridabad, Haryana,INDIA \\ sanc_1988@yahoo.com
}

\begin{abstract}
In a biometric system a person is identified automatically by processing the unique features that are posed by the individual. Iris Recognition is regarded as the most reliable and accurate biometric identification system available. In Iris Recognition a person is identified by the iris which is the part of eye using pattern matching or image processing using concepts of neural networks. The aim is to identify a person in real time, with high efficiency and accuracy by analysing the random patters visible within the iris if an eye from some distance, by implementing modified Canny edge detector algorithm. The major applications of this technology so far have been: substituting for passports (automated international border crossing); aviation security and controlling access to restricted areas at airports; database access and computer login.
\end{abstract}

Keywords - iris recognition, biometric identification, pattern recognition, segmentation

\section{INTRODUCTION}

Iris recognition is the process of recognizing a person by analyzing the random pattern of the iris (Figure 1). The automated method of iris recognition is relatively young, existing in patent only since 1994.The iris is a muscle within the eye that regulates the size of the pupil, controlling the amount of light that enters the eye. It is the coloured portion of the eye with colouring based on the amount of melatonin pigment within the muscle (Figure 2).[2]

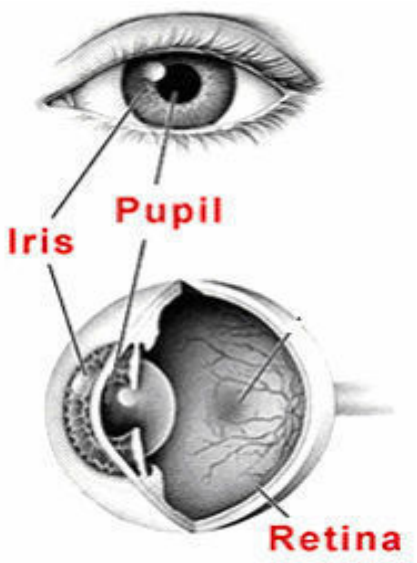

Figure.1 Human Eye

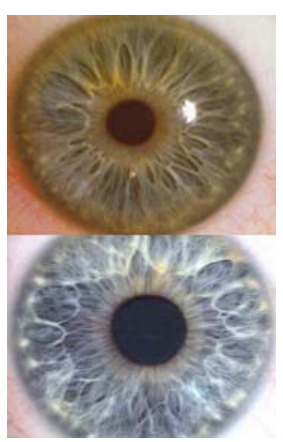

Figure.2 Colored porton of eye

\section{II .THE IRIS AS A BIOMETRICS}

The iris is an overt body that is available for remote assessment with the aid of a machine vision system to do automated iris recognition.

A. Iris recognition technology combines computer vision, pattern recognition, statistical inference, and optics.

B. The spatial patterns that are apparent in the human iris are highly distinctive to an individual.

- Clinical observation

- Developmental biology

Although the coloration and structure of the iris is genetically linked, the details of the pattern are not. The iris develops during prenatal growth through a process of tight forming and folding of the tissue membrane. Prior to birth, degeneration occurs, resulting in the pupil opening and the random, unique patterns of the iris. Although genetically identical, an individual's irides are unique and structurally distinct, which allows for it to be used for recognition purposes. [1][2]

\section{METHODOLOGY}

The system is to be composed of a number of sub-systems, which correspond to each stage of iris recognition. These stages are: 
- Image acquisition-capturing eye image

- segmentation - locating the iris region in an eye image

- $\quad$ normalization - creating a dimensionally consistent representation of the iris region

- feature encoding - creating a template containing only the most discriminating features of the iris.[9][2]

The input to the system will be an eye image, and the output will be an iris template, which will provide a mathematical representation of the iris region.[2]

\section{IMAGE ACQUISITION}

The iris image should be rich in iris texture as the feature extraction stage depends upon the image quality. Thus, the image is acquired by 3CCD camera placed at a distance of approximately $9 \mathrm{~cm}$ from the user eye. The approximate distance between the user and the source of light is about 12 $\mathrm{cm}$. The image acquisition setup is given in Figure 1. The following attentions have been taken care at the time of grabbing the image

- High resolution and good sharpness: It is necessary for the accurate detection of outer and inner circle boundaries

- Good lighting condition: The system of diffused light is used to prevent spotlight effect[10]

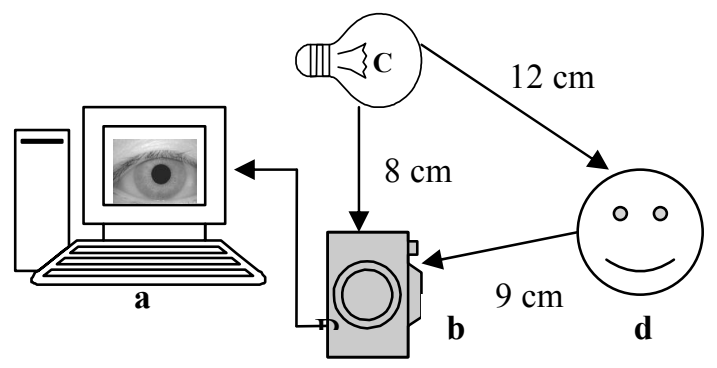

Figure3. Image acquisition System (a) System with frame grabber (b)

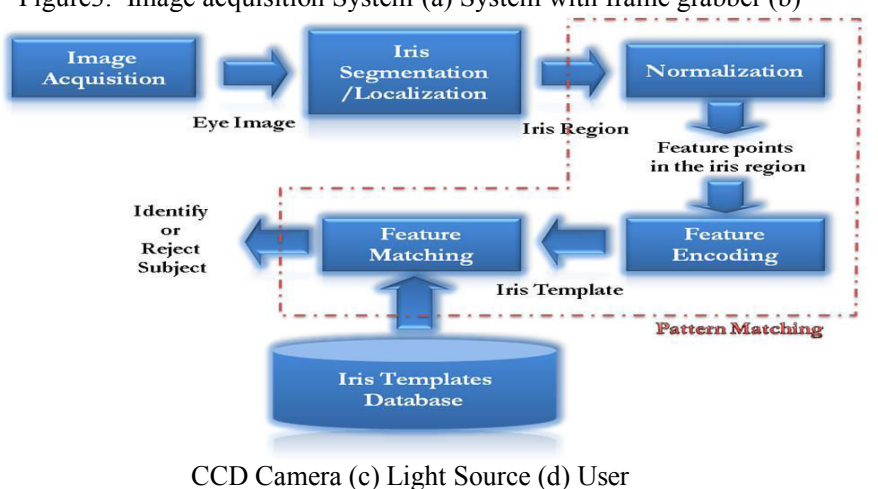

Figure4. Schematic diagram of iris recognition

\section{SEGMENTATION}

The first stage of iris recognition is to isolate the actual iris region in a digital eye image. The iris region, shown in the above figure, can be approximated by two circles, one for the iris/sclera boundary and another, interior to the first, for the iris/pupil boundary.[5][6]

The success of segmentation depends on the imaging quality of eye images. The center of pupil can be used to detect the outer radius of iris patterns. The iris inner and outer boundaries are located by finding the edge image using the Canny edge detector [6].

\section{MODIFIED CANNY EDGE DETECTOR}

The algorithm runs in 5 separate steps:

1. Smoothing: Filtering and blurring of the image to remove noise, such that pixels creating indifferent spots can be reduced.

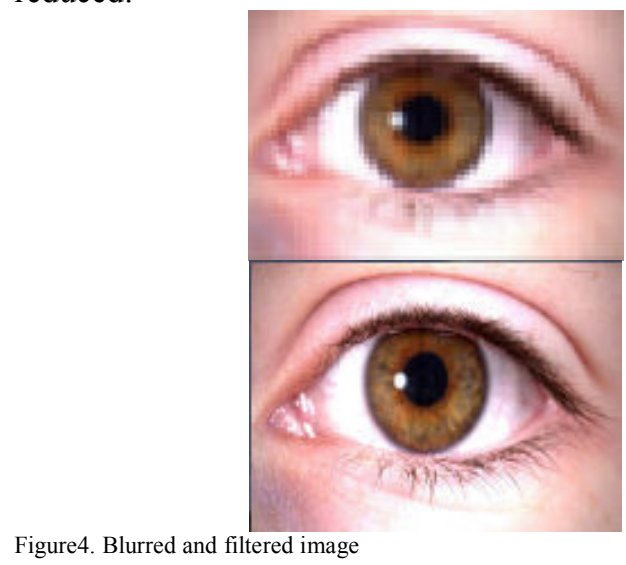

2. Finding gradients: At the points/pixels where colour pattern falls in the similar threshold region are grouped together.

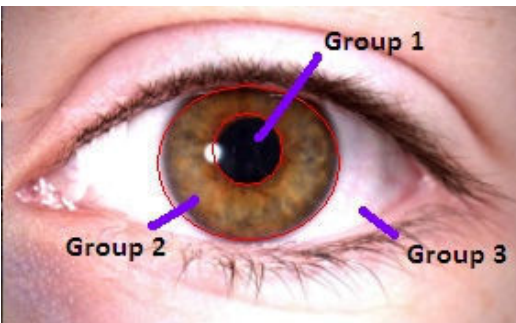

Figure 5 Grouping of various regions of eye on the basis of colour differences.

The edges should be marked where the gradients of the image has large magnitudes.

3. Non-maximum suppression: The image portion to be processed is non linear and circular or convex hence, boundary region matching the closets shape is taken out for only local maxima and then should be marked as edges. 


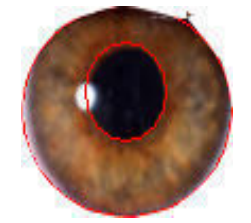

Figure6. Crop out portion of eye that needs processing.

4. Double thresholding: Potential edges are determined by thresholding.

5. Edge tracking by hysteresis: Final edges are determined by suppressing all edges that are not connected to a very certain (strong) edge.[12]

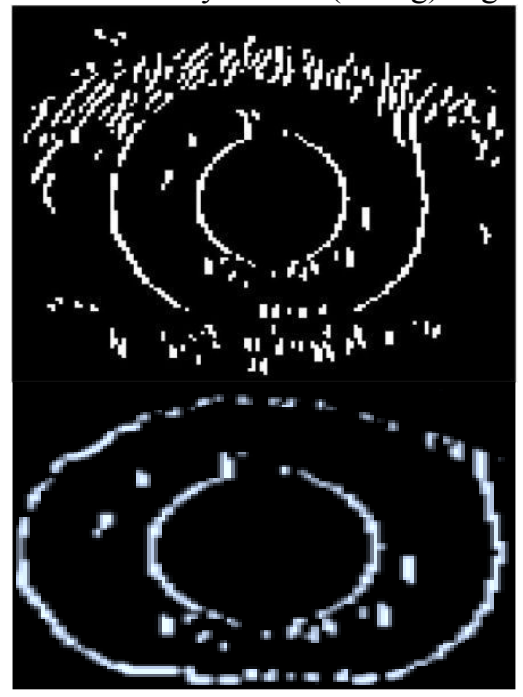

Fig-7. Canny edge image with result of modified image using algorithm.

\section{HOUGH TRANSFORM}

The Hough transform is a standard computer vision algorithm that can be used to determine the parameters of simple geometric objects, such as lines and circles, present in an image. The circular Hough transform can be employed to deduce the radius and centre coordinates of the pupil and iris regions.[1][7]

Firstly, an edge map is generated by calculating the first derivatives of intensity values in an eye image and then thresholding the result. From the edge map, votes are cast in Hough space for the parameters of circles passing through each edge point. These parameters are the centre coordinates $x$ and $y$, and the radius $r$, which are able to define any circle according to the equation

$$
\mathrm{x}^{2} \mathrm{C}+\mathrm{y} 2_{\mathrm{C}-\mathrm{r}^{2}}=0
$$

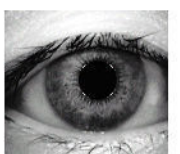

(a)

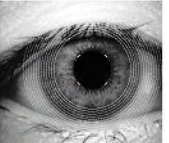

(b)

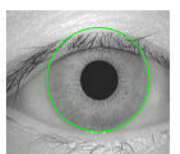

(c)
Figure 8 (a) Contrast enhanced image

(b) Concentric circles of different radii

(c) Localized Iris image

\section{IMAGE NORMALIZATION}

Once the iris region is segmented, the next stage is to normalize this part, to enable generation of the iris code and their comparisons. Since variations in the eye, like optical size of the iris, position of pupil in the iris, and the iris orientation change person to person, it is required to normalize the iris image, so that the representation is common to all, with similar dimensions.[8]

Normalization process involves unwrapping the iris and converting it into its polar equivalent. It is done using Daugman's Rubber sheet model. The centre of the pupil is considered as the reference point and a Remapping formula is used to convert the points on the Cartesian scale to the polar scale.

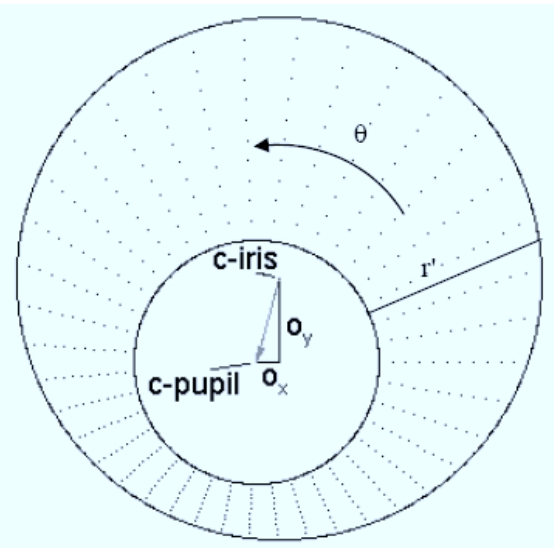

Figure 9: Normalization process

$$
r^{\prime}=\sqrt{\alpha} \beta \pm \sqrt{\alpha \beta^{2}-\alpha-r_{I}^{2}}
$$

Where $\mathrm{r} 1=$ iris radius

$$
\begin{aligned}
& \alpha=o_{x}^{2}+o_{y}^{2} \\
& \beta=\cos \left(\pi-\arctan \left(\frac{o_{y}}{o_{x}}\right)-\theta\right)
\end{aligned}
$$

The radial resolution was set to 100 and the angular resolution to 2400 pixels. For every pixel in the iris, an equivalent position is found out on polar axes.[8]The normalized image was then interpolated into the size of the original image, by using the interp2 function. The parts in 
the normalized image which yield a $\mathrm{NaN}$, are divided by the sum to get a normalized value.[8][5]

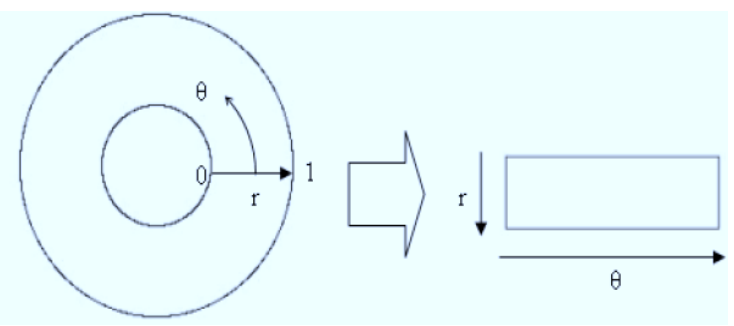

Figure 8 : Unwrapping the iris

\section{ENCODING}

The final process is the generation of the iris code. For

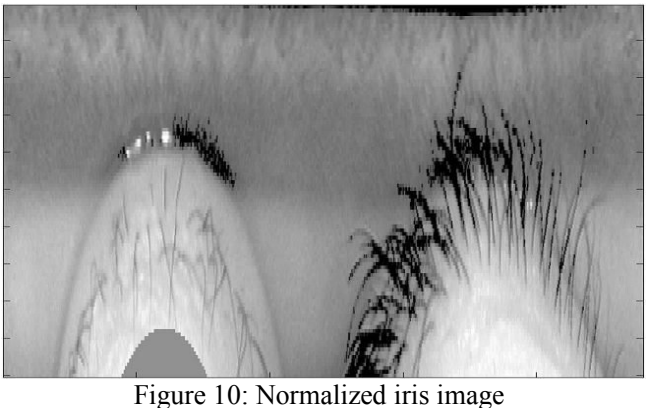

this, the most discriminating feature in the iris pattern is extracted. The phase information in the pattern only is used because the phase angles are assigned regardless of the image contrast.[10] Amplitude information is not used since it depends on extraneous factors. Extraction of the phase information, according to Daugman, is done using 2D Gabor wavelets. It determines which quadrant the resulting phasor lies using the wavelet:

$$
\begin{array}{r}
h_{\{R e, I m\}}=\operatorname{sgn}_{\{R e, I m\}} \int_{\rho} \int_{\phi} I(\rho, \phi) e^{-i \omega\left(\theta_{0}-\phi\right)} \\
\cdot e^{-\left(r_{0}-\rho\right)^{2} / \alpha^{2}} e^{-\left(\theta_{0}-\phi\right)^{2} / \beta^{2}} \rho d \rho d \phi
\end{array}
$$

where, $\left.h_{\{R e, I m}\right\}$ has the real and imaginary part, each having the value 1 or 0 , depending on which quadrant it lies in.

An easier way of using the Gabor filter is by breaking up the $2 \mathrm{D}$ normalized pattern into a number of $1 \mathrm{D}$ wavelets, and then these signals are convolved with 1D Gabor wavelets.[8]

Gabor filters are used to extract localized frequency information. But, due to a few of its limitations, log-Gabor filters are more widely used for coding natural images. It was suggested by Field, that the log filters (which use Gaussian transfer functions viewed on a logarithmic scale) can code natural images better than Gabor filters (viewed on a linear scale). Statistics of natural images indicate the presence of high-frequency components.[1][8] Since the ordinary Gabor filters under-represent high frequency components, the log filters become a better choice. LogGabor filters are constructed using

$$
G(f)=\exp \left(\frac{-\left(\log \left(f / f_{0}\right)\right)^{2}}{2\left(\log \left(\sigma / f_{0}\right)\right)^{2}}\right)
$$

\section{PATTERN MATCHING}

- Purpose: to establish a precise correspondence between characteristic structures across the two images.

- Both of the systems under discussion compensate for image shift, scaling, and rotation.

- For both systems, iris localization is charged with isolating an iris in a larger acquired image and thereby accomplishes alignment for image shift.

In pattern matching of pixels with the databases will be done using the following algorithm:

An emerging technique in this particular application area is the use of Artificial Neural Network implementations with networks employing specific guides (learning rules) to update the links (weights) between their nodes. Such networks can be fed the data from the graphic analysis of the input picture and trained to output characters in one or another form. Specifically some network models use a set of desired outputs to compare with the output and compute an error to make use of in adjusting their weights. Such learning rules are termed as Supervised Learning.

\section{BACK PROPAGATION}

Back propagation, or propagation of error, is a common method of teaching artificial neural networks how to perform a given task. It requires a teacher that knows, or can calculate, the desired output for any given input. It is most useful for feed-forward networks (networks that have no feedback, or simply, that have no connections that loop).[13] The term is an abbreviation for "backwards propagation of errors". Back propagation requires that the activation function used by the artificial neurons (or "nodes") is differentiable. It has two phases:

\section{Training}

\section{Testing}




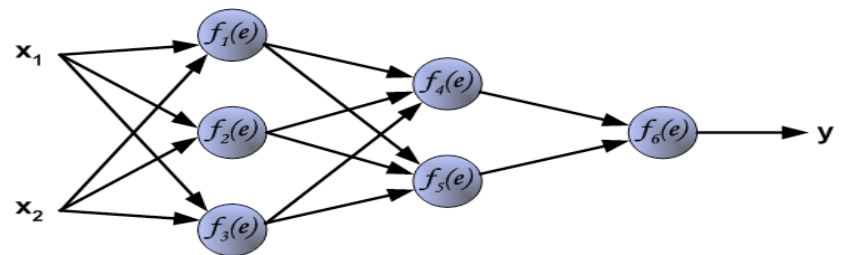

Figure11. Three layer neural network with two inputs and one output

Each neuron is composed of two units. First unit adds products of weights coefficients and input signals. The second unit realise nonlinear function, called neuron activation function. Signal $e$ is adder output signal, and $y=f(e)$ is output signal of nonlinear element. Signal $y$ is also output signal of neuron.
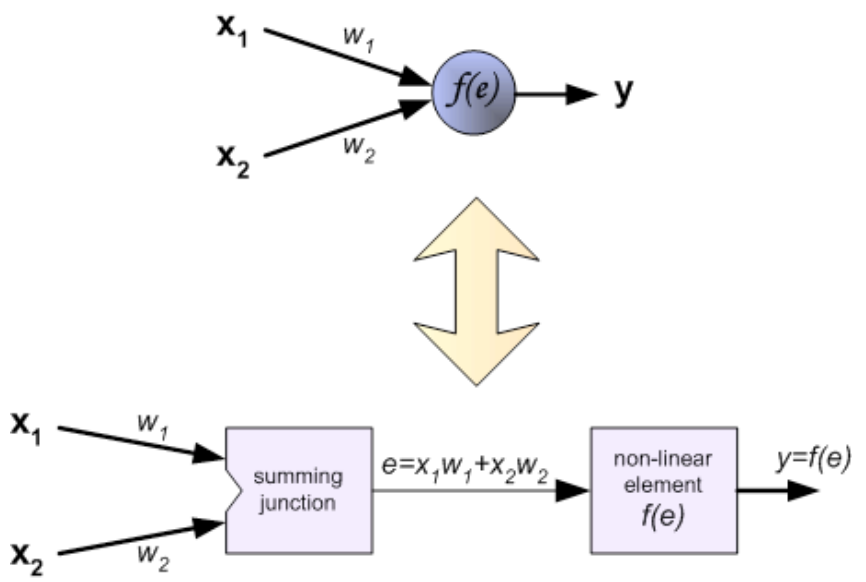

Figure12 Functioning of system with weights.

To teach the neural network we need training data set. The training data set consists of input signals ( $x_{1}$ and $\left.x_{2}\right)$ assigned with corresponding target (desired output) $z$.

The first method is to start teaching process with large value of the parameter. While weights coefficients are being established the parameter is being decreased gradually. The second, more complicated, method starts teaching with small parameter value.[13]

\section{Back propagation Algorithm}

Actual algorithm for a 3-layer network (only one hidden layer):

Initialize the weights in the network (often randomly)
Do

For each example e in the training set

$\mathrm{O}=$ neural-net-output(network, e) ; forward pass

$\mathrm{T}=$ teacher output for $\mathrm{e}$

Calculate error $(\mathrm{T}-\mathrm{O})$ at the output units

Compute delta_wh for all weights from hidden

layer to output layer ; backward pass

Compute delta_wi for all weights from input layer to hidden layer; backward pass continued

Update the weights in the network

Until all examples classified correctly or stopping criterion satisfied Return the network[14]

\section{APPLICATIONS}

- Today's e-security is in critical need of finding accurate, secure and cost-effective alternatives to passwords and personal identification numbers (PIN) as financial losses increase dramatically year over year from computer-based fraud such as computer hacking and identity theft . [3]

- Biometric solutions address these fundamental problems, because an individual's biometric data is unique and cannot be transferred and therefore can be used for identifying a person or verifying the identity of a person.

- For an enterprise, biometrics provides value in two ways. First, a biometric device automates entry into secure locations, relieving or at least reducing the need for full-time monitoring by personnel. Second, when rolled into an authentication scheme, biometrics adds a strong layer of verification for user names and passwords.

- Biometrics adds a unique identifier to network authentication, one that is extremely difficult to duplicate. Smart cards and tokens also provide a unique identifier, but biometrics has an advantage over these devices.

- It is being implemented and substituted for passports (automated international border crossing), aviation security and controlling access to restricted areas at airports, database access and computer login, premises access control.[3] 


\section{WORK CITED}

We have developed this recognition system as our major project in our final year. In the project we used canny edge and Hough transform algorithms to find the iris region. After that we applied the Daugman's algorithm to convert the circular region into rectangular block.

Backpropogation algorithm was developed on our own in which we developed a network taking input images as the normalised images obtained from normalization process.

One of the images from the database was used as the main image for iris comparison. In testing phase we tested whether the main image was there in our database or not using the trained algorithm. If the image was found then the recognition system was a success otherwise backpropogation would start training the network again.

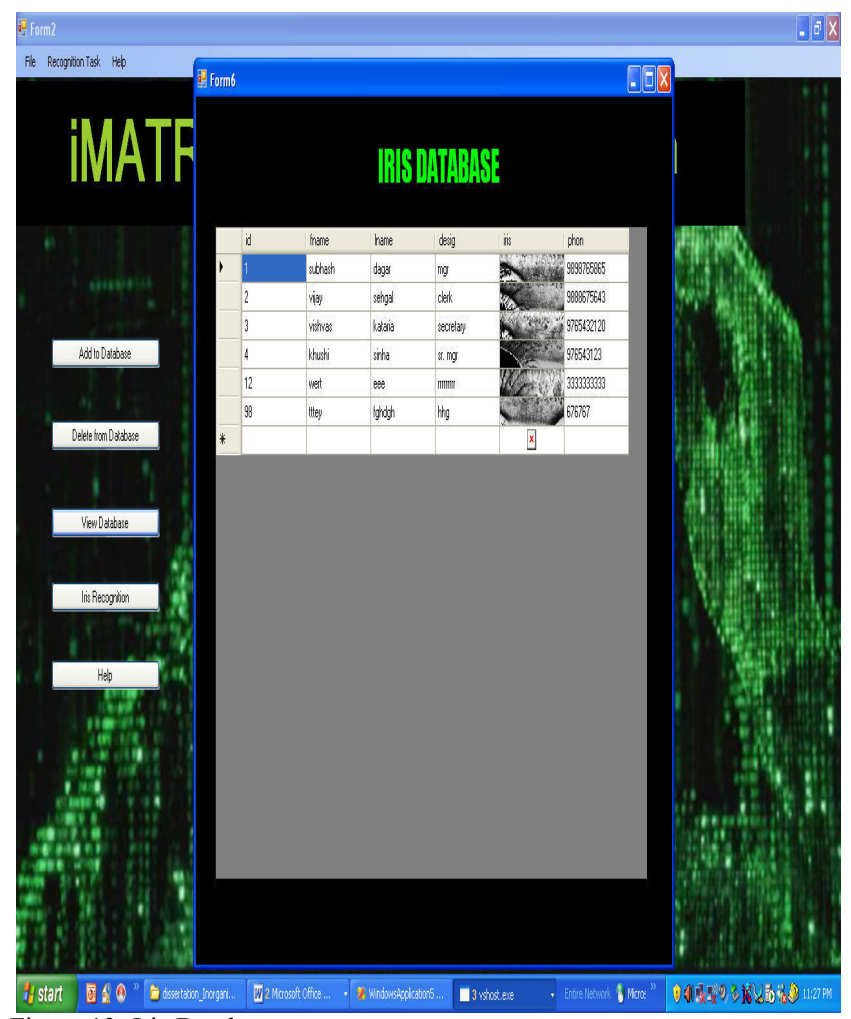

Figure 13: Iris Database

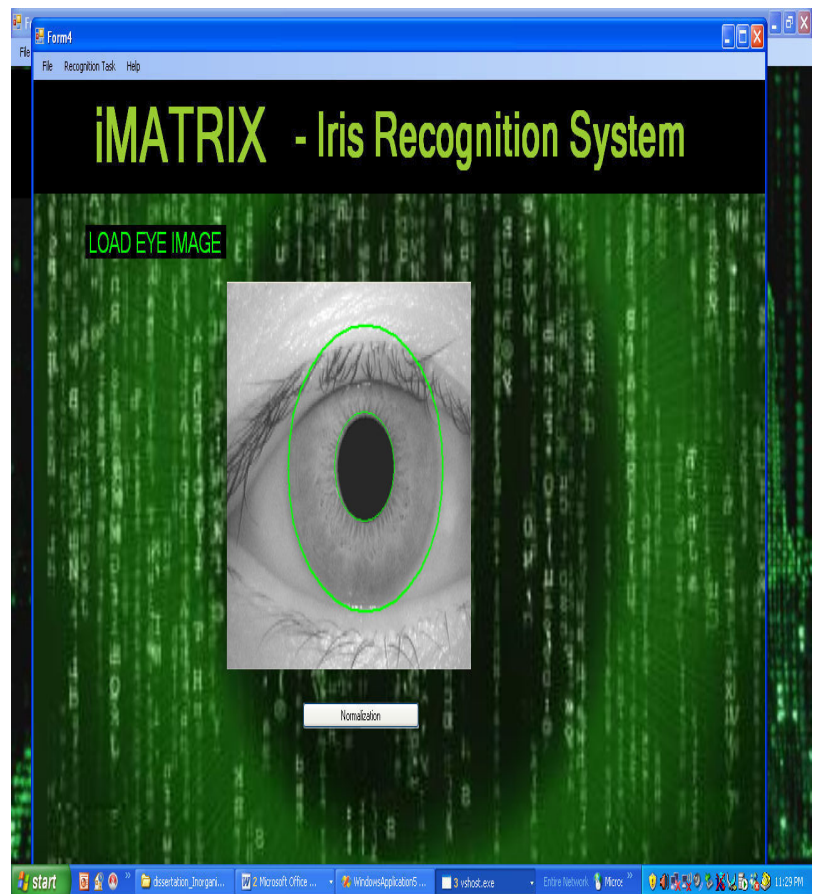

Figure 14: Loading image from data base

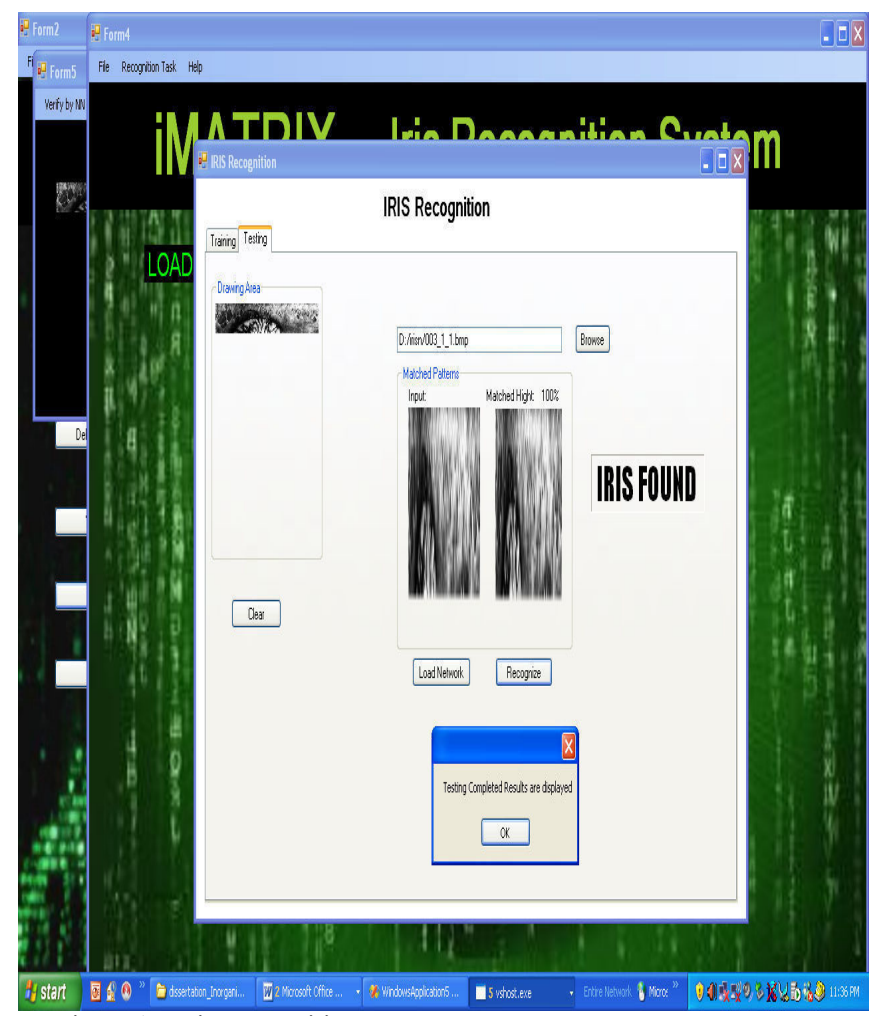

Figure 15: Iris Recognition 


\section{CONCLUSION}

In this paper we have analysed how the network behaves when an input is given and for that error rate specified was. The network has been trained and tested for a number of eye images. our project is a system that can take a image(as input of human eye) and can distinguish between pupillary body and iris part of the human eye. For this we had used different mathematical functions and calculations to detect various eye boundaries and it encircles outer boundary of pupil which is inner boundary for the iris using modified Canny edge detector algorithm. After this the detection of outer boundary of the iris is done. The development tool used is c\# using windows application, matlab and emphasis is given on software for performing recognition, and not hardware for capturing an eye image.

\section{A. Performance Observation.}

1. Increasing the number of images in the training network generally slowed down the learning rate.

2. The size of the input states is also another direct factor influencing the performance. It is natural that the more number of input symbol set the network is required to be trained for the more it is susceptible for error.

\section{REFERENCES}

[1]http://www.csse.uwa.edu.au/ pk/studentprojects/libor/in dex.html

[2].http://www.biometricscatalog.org/NSTCSubcommittee/

Documents/Iris\%20Recognition.pdf

[3]. John.Daugman@CL.cam.ac.uk

[4]. J. G. Daugman: High confidence visual recognition of persons by a test of statistical independence. IEEE Transactions on Pattern Analysis and Machine Intelligence Vol. 15 (1993) 1148-1161

[5]..W.W. Boles, B. Boashah: A Human Identification Technique Using Images of the Iris and Wavelet Transform. IEEE Transaction on Signal Processing Vol. 46 (1998) 1185-1188

[6]. T. Chuan Chen, K. Liang Chung: An Efficient Randomized Algorithm for Detecting Circles.

Computer Vision and Image Understanding Vol. 83 (2001) 172-191

[7]. T. Chuan Chen, K. Liang Chung: An Efficient Randomized Algorithm for Detecting Circles.

Computer Vision and Image Understanding Vol. 83 (2001) 172-191

[8]. Peter Kovesi, Matlab functions for Computer Vision and Image Processing. What are Log-Gabor filters?
[9].xpertsolutions.fortunecity.es/Technology/Biometrics/Xp recEng.htm

[10].L. Flom and A. Safir: Iris Recognition System. U.S. atent No.4641394 (1987)

[11]. E. R. Davies: Machine Vision. $3^{\text {rd }}$ Edition: Elsevier (2005)

[12]. J. Canny: A Computational Approach to Edge Detection. IEEE Transaction on Pattern Analysis and Machine Intelligence Vol. 8 (1986) 679-714

[13]Ryszard Tadeusiewcz "Sieci neuronowe", Kraków 1992 [14]. http://page.mi.fu-berlin.de/rojas/neural/chapter/K7.pdf

\section{AUTHORS PROFILE}

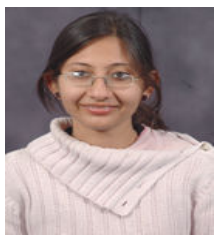

Neha Kak, a final year Computer Science student at Lingaya's Institute of Mngt. \& Tech., Faridabad, Haryana, India. Areas of interests include DBMS, data warehouse and data mining, Web Technologies.

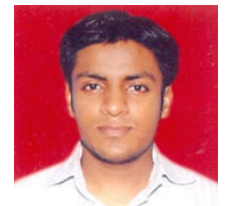

Rishi Gupta, a final year Computer Science student at Lingaya's Institute of Mngt. \& Tech., Faridabad, Haryana, India. Subjects under study includes Computer networks, operating systems, Image processing.

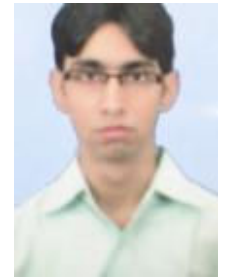

Sanchit Mahajan, a final year information technology student at Lingaya's Institute of Mngt. \& Tech., Faridabad, Haryana, India. Subjects are DBMS, CN, OS, Image processing, A.I. 